\title{
Hybrid Medical Imaging: Scanning Thermoacoustic Tomography
}

\author{
Lihong V. Wang and Geng Ku \\ Optical Imaging Laboratory, Biomedical Engineering Program, \\ Texas A\&M University, 3120 TAMU \\ College Station, TX 77843-3120 \\ LWang@tamu.edu \& http://oilab.tamu.edu
}

\begin{abstract}
Scanning thermoacoustic tomography based on microwave-induced thermoacoustic waves was studied. Two-dimensional images of 50-mm thick biological tissue samples were obtained experimentally. The thermoacoustic signals were also simulated theoretically. The image resolution was significantly improved compared with purely microwave imaging.

Keywords: microwave, ultrasonics, thermoacoustics, photoacoustics, tomography.
\end{abstract}

\section{Introduction}

Microwave imaging of biological tissues has been investigated for a number of years [1,2]. The advantages of the technique include the use of non-ionizing radiation and high imaging contrast. However, the technique has had difficulties primarily in the reconstruction algorithms and in achieving good spatial resolution. Ultrasound imaging (ultrasonography), an established medical imaging modality, can yield good spatial resolution but has poor contrast. Microwaveinduced acoustics (MIA) may bridge the gap and combine the advantages of the two types of radiation. MIA was also employed by several investigators in the 1980s for imaging of biological tissues or tissue phantoms $[3,4,5]$. However, these early works did not produce any tomographic or depth-resolved images. Recently, images of biological tissue were reconstructed based on MIA [6]. This approach requires measurements of a large amount of data around the tissue and computation following the data acquisition.

We present our study on microwave-induced scanning thermoacoustic tomography (STAT) toward biomedical applications $[7,8]$. The microwave-induced acoustic signal was simulated to explain the experimental results. Two-dimensional cross sections of biological tissues were imaged using STAT. The presented imaging approach here differs significantly from the prior arts. Lateral resolution was achieved by the use of a focused ultrasonic transducer. Axial resolution was obtained by measuring the temporal profiles of the microwave-induced acoustic signals. Depth-resolved tomographic images were acquired directly without resorting to image reconstruction.

\section{Experimental setup}

The experimental setup used for this study is shown in Fig. 1. A Cartesian coordinate system was set up for reference. The $z$ axis was along the ultrasonic axis pointing upward. The $x$ axis was perpendicular to the drawing plane and pointed outward. The $y$ axis was in the drawing plane pointing to the right. A $3-\mathrm{GHz}$ pulse microwave generator transmitted 
microwave pulses. A function generator (DS345, Stanford Research System) was employed to trigger the microwave generator, control its pulse repetition frequency and synchronize the oscilloscope sampling. Microwave energy was delivered by a waveguide. The biological sample was fixed on a two-dimensional $x$ - $y$ translation stage (MD2, Arrick Robotics). A personal computer controlled the two stepper motors to drive the translation stage in the $x$ and $y$ directions. An ultrasonic transducer (UST) was in contact with

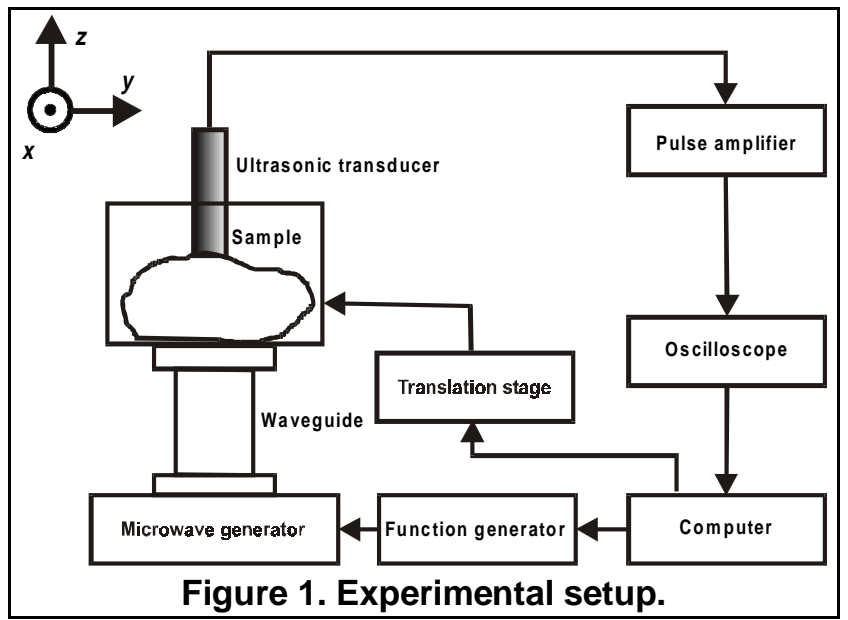

Figure 1. Experimental setup. the sample. Two ultrasonic transducers were used in this study. For the first one (V314, Panametrics), the central frequency of the ultrasonic transducer was $1 \mathrm{MHz}$, the $-6 \mathrm{~dB}$ bandwidth was $0.65 \mathrm{MHz}$, the diameter was $1.9 \mathrm{~cm}$, and the focal length at $1 \mathrm{MHz}$ was $2.5 \mathrm{~cm}$. For the second one (V384, Panametrics), the central frequency was $3.5 \mathrm{MHz}$, the $-6 \mathrm{~dB}$ bandwidth was $2.5 \mathrm{MHz}$, the diameter was $0.64 \mathrm{~cm}$, and the focal length at $3.5 \mathrm{MHz}$ was 1.8 $\mathrm{cm}$. The transducer was connected to a pulse amplifier with a $25-\mathrm{MHz}$ bandwidth (500PR, Panametrics). The amplified signal was recorded by an oscilloscope (TDS-640A, Tektronix) and transferred to a personal computer.

\section{Results and Discussion}

The generation of thermoacoustic waves by deposition of microwave energy can be described by the following differential equation:

$$
\left(\nabla^{2}-\frac{1}{v_{s}^{2}} \frac{\partial^{2}}{\partial t^{2}}\right) p(\mathbf{r}, t)=-\frac{\beta}{C_{p}} \frac{\partial H}{\partial t},
$$

where $p(\mathbf{r}, t)$ is the thermoacoustic pressure at the position $\mathbf{r}$ and time $t, v_{s}$ is the speed of sound, $\beta$ is the isobaric volume expansion coefficient, $C_{p}$ is the heat capacity, and $H$ is the heating function describing the microwave-energy deposition in the sample per unit volume per unit time. Thermal-confinement condition is assumed, where the acoustic transit time across the acoustic source is less than the heat conduction time. The solution of the three-dimensional wave equation under the zero-initial-value conditions $p(0, \mathbf{r})=0$ and $\frac{\partial}{\partial t} p(0, \mathbf{r})=0$ can be expressed as an integral:

$$
p(\mathbf{r}, t)=\frac{\beta}{4 \pi C_{p}} \iiint \frac{1}{\left|\mathbf{r}-\mathbf{r}^{\prime}\right|} \frac{\partial H\left(\mathbf{r}^{\prime}, t^{\prime}\right)}{\partial t^{\prime}} d \mathbf{r}^{\prime} .
$$

The integral is calculated inside a sphere with a radius of $v_{s} t$ centered at $\mathbf{r}$, and $\mathbf{r}^{\prime}$ is the position inside the sphere where microwave is absorbed and acoustic signal is generated. In the integration, the heating function is not taken at time $t$ but at an earlier time $t^{\prime}=t-\left|\mathbf{r}-\mathbf{r}^{\prime}\right| / v_{s}$; therefore, the integration function is also called retarded potential [9]. Analytic solutions can be obtained for simple geometric structures such as an infinite layer, a sphere, and a cylinder under delta heating, where the heating function is a delta function in time [10].

For a slab with a thickness $d$ under delta heating, the impulse-response pressure is 


$$
p_{1}(z, t)=\frac{\beta v_{s}^{2}}{2 C_{p}} u\left(z-v_{s} t\right),
$$

where $u\left(z-v_{s} t\right)$ is defined as a function that is unity when $0 \leq\left(z-v_{s} t\right) \leq d$ and zero otherwise. The impulse response is a traveling square wave as shown in Fig. 2(a) for a 4.8-mm gel slab if the microwave attenuation across the slab is negligible. Because the propagation speed of electromagnetic wave is much greater than the speed of sound, the sample volume illuminated by microwave pulses radiates acoustic waves simultaneously.

The heating function in one-dimensional lossy media may be expressed as $H(z, t)=\alpha \exp (-\alpha z) s(t)$, where $\alpha$ is the microwave absorption coefficient, and $s(t)$ is the temporal profile of the microwave pulse. Figure 2(b) shows the temporal profile of the microwave pulses used in our experiment.

The thermoacoustic pressure induced by the microwave pulses can be derived by the following convolution:

$$
p(z, t)=\int p_{1}(z, \tau) H(z, t-\tau) d \tau .
$$

Figure 2(c) illustrates the thermoacoustic pressure at the ultrasonic transducer generated from the slab, which was obtained by convoluting the two temporal waveforms in Figs. 2(a) and 2(b).

For more general non-thermal-confined cases when heat transfer in the medium cannot be neglected, the following heat conduction equation must be taken into account in the pressure calculation:

$$
\rho C_{p} \frac{\partial T(\mathbf{r}, t)}{\partial t}=k \nabla^{2} T(\mathbf{r}, t)+H(\mathbf{r}, t),
$$

where $\rho$ is the density of the medium, $k$ is the thermal conductivity, and $T(\mathbf{r}, t)$ is the temperature distribution in the microwave-illuminated space. The thermoacoustic pressure is:

$$
p(r, t)=\frac{\beta}{4 \pi C_{p}} \iiint \frac{1}{\left|r-r^{\prime}\right|}\left[\frac{\partial H\left(r^{\prime}, t^{\prime}\right)}{\partial t^{\prime}}+k \nabla^{2} \frac{\partial T\left(r^{\prime}, t\right)}{\partial t^{\prime}}\right] d r^{\prime} .
$$

The integration involves the heat conducted from the surrounding medium besides the heat due to the absorbed microwave energy.

The experimentally measured piezo-electric impulse response $q(t)$ of the ultrasonic transducer is shown in Fig. 2(d). The piezo-electric output of the ultrasonic transducer in response to thermoacoustic pressure can be calculated by the following convolution between the thermoacoustic pressure at the transducer and the impulse response of the transducer:

$$
P_{o}(z, t)=\int p(z, \tau) q(\tau-t) d \tau \text {. }
$$

The piezo-electric signal from a $4.8-\mathrm{mm}$ slab was obtained by the convolution of the two waveforms in Figs. 2(c) and 2(d) with Eq. (7) and is plotted in Fig. 2(e) as a dashed line.

The acoustic signal generated by the microwave pulse and detected by the ultrasonic transducer can be simulated by two convolutions: 1) convolution between the thermoacoustic signal of an ideal delta-pulse microwave and the actual microwave pulse, 2) further convolution with the impulse response of the UST. The pulse width of the temporal thermoacoustic signal is approximately the sum of the microwave pulse width and the response time of the detection system. The simulated result was shown as Fig. 2. 


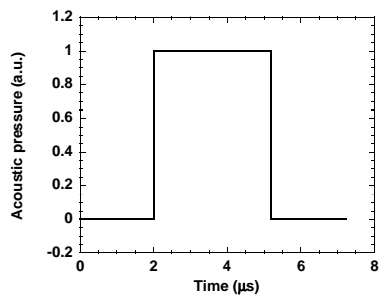

(a)

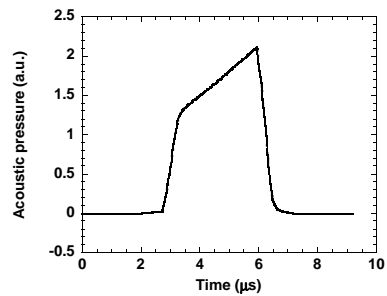

(c)

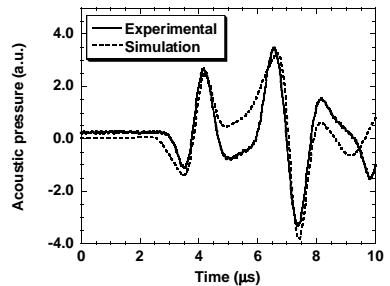

(e)

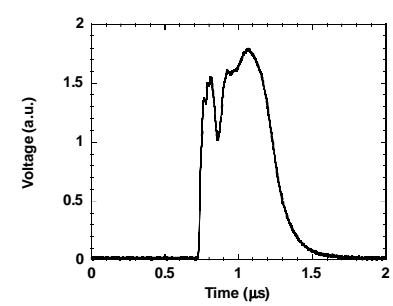

(b)

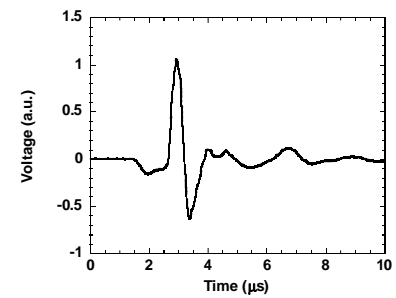

(d)

Figure 2. Simulation of the piezo-electric signal in response to the microwaveinduced thermoacoustic signal from a 4.8-mm slab sample. (a) Thermoacoustic signal in a slab to a microwave impulse; (b) Temporal profile of the microwave pulses used in the experiment;

Thermoacoustic signal in the slab induced by the microwave pulses used in the experiment, electrical field attenuation was considered; (d) Piezo-electric impulse response of the ultrasonic transducer; (e) Simulated and experimental piezo-electric outputs of the ultrasonic transducer in response to the thermoacoustic signals.

We detected dipolar structures in the thermoacoustic signals in slabs resulted from the limited bandwidth of the ultrasonic transducers. This phenomenon can also be explained clearly in the frequency domain, where the ultrasonic transducer acts as a band-pass filter. A frequency spectrum of the ultrasonic transducer is shown in Fig. 3(a). The temporal profile of the thermoacoustic pressure varies sharply near the slab boundaries and slowly inside the slab as shown in Fig. 2(c). The corresponding spectrum is peaked at DC as shown in Fig. 3(b). The filtered spectrum is peaked near $0.5 \mathrm{MHz}$ as shown in Fig. 3(c), where the DC is rejected and the low-frequency components are attenuated significantly. In other words, the ultrasonic transducer cannot respond efficiently to the thermoacoustic waves emitted between the sample boundaries, which have a lower-frequency spectrum. Therefore, the observed piezo-electric signal between the two sample boundaries is low.

The UST detector measured the time of arrival of the thermoacoustic waves. The distance between the acoustic sources and the transducer was calculated by multiplying the time of arrival with the speed of sound in the medium. Therefore, a time-domain signal could be converted into a one-dimensional image along the transducer axis ( $z$ axis). A sample of a fatmuscle-fat structure shown in Fig. 4(a) was imaged. Scanning the sample along the $x$ or $y$ axis yielded a two-dimensional cross-sectional image of the sample. Figure 4(b) shows a 2D-image of the fat-muscle-fat structure obtained with the 1-MHz UST. The thermoacoustic signals in the time domain were acquired while the fat-muscle-fat sample was scanned horizontally along the $y$ axis with a step size of $1 \mathrm{~mm}$. Each vertical line in this $2 \mathrm{D}$ image was obtained from a temporal thermoacoustic waveform. 


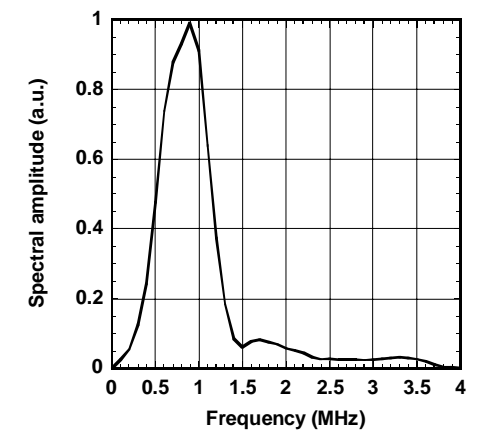

(a)

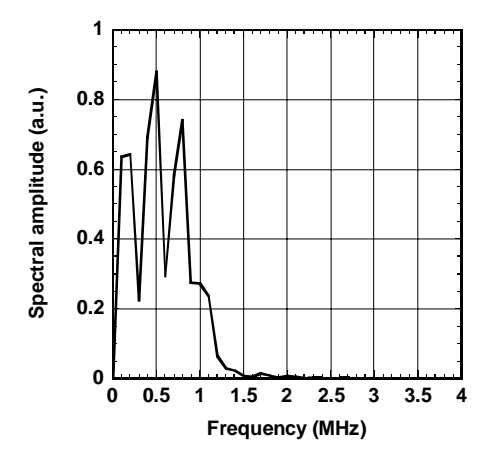

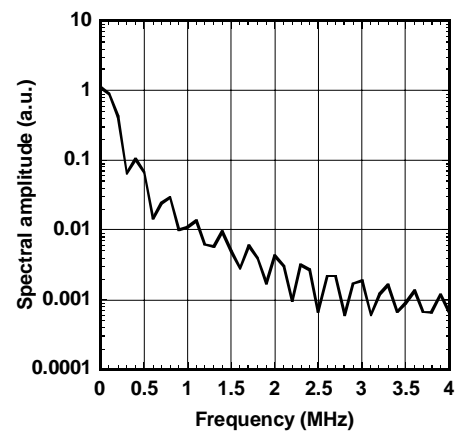

(b)

(c)

Figure 3. Frequency-domain analysis of the microwave-induced thermoacoustic signals. (a) Spectrum of the 1-MHz ultrasonic transducer; (b) Spectrum of the microwave-induced thermoacoustic signal; (c) Spectrum of the piezo-electric signal, which was the filtered microwave-induced thermoacoustic signal.

The axial resolution along the UST axis ( $z$ axis) is determined by the pulse width of the microwave pulse and the bandwidth of the UST. When the $1-\mathrm{MHz}$ ultrasonic transducer was used, the width of the thermoacoustic signal was estimated to be $2.2 \mu \mathrm{s}$, which was the sum of the width of the microwave pulses $(0.5 \mu \mathrm{s})$ and the width of the impulse response of the transducer $(1.7 \mu \mathrm{s})$. Because the speed of sound in tissue is $\sim 1.5 \mathrm{~mm} / \mu \mathrm{s}$, the corresponding axial resolution was $\sim 3.3 \mathrm{~mm}$ along the $z$ axis. For the $3.5-\mathrm{MHz}$ ultrasonic transducer, the axial resolution can be improved to $\sim 1.4 \mathrm{~mm}$. This resolution is considerably better that the resolution that can be achieved in purely microwave imaging $(>5 \mathrm{~mm})$, which is approximately one half of the microwave wavelength. The focused UST detector responded the thermoacoustic source in its focal zone. Because the focal diameter and the focal zone are frequency dependent, the lateral resolution is affected by the spectrum of the thermoacoustic source. The spectrum of acoustic emission stimulated by the microwave pulses concentrated mainly at lower frequencies. The spectral intensity decreased as the frequency increased. The detected area under the UST was larger than that estimated by the central frequency of the UST. When the $3.5-\mathrm{MHz}$ UST with $18-\mathrm{mm}$ focal length was used as the detector, the lateral resolution reached $2 \mathrm{~mm}$. 


\section{Conclusion}

Our studies show that scanning microwave-induced thermoacoustic tomography is a promising imaging tool for biological tissue. The boundaries between different tissue constituents can be imaged clearly. The width of the microwave pulses and the bandwidth of the ultrasonic transducer determine the axial resolution along the acoustic axis of the ultrasonic transducer. The axial resolution can be improved by employing even shorter microwave pulses and broader-band ultrasonic transducers.

\section{Acknowledgments}

This project was sponsored in part by the National Institutes of Health grants R29 CA68562, R01 CA71980, and R21 CA83760 and by the National Science Foudation grant BES-9734491.
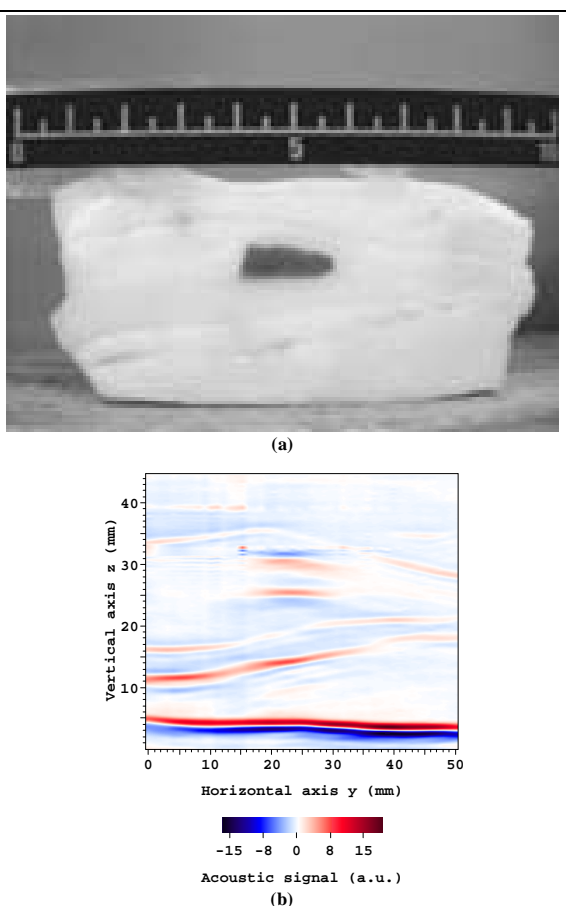

Figure 4. (a) Cross section of a sample consisting of fat and muscle tissues; (b) A 2D image of the sample.

\section{References}

1 L. E. Larsen and J. H. Jacobi, eds., Medical Applications of Microwave Imaging (IEEE Press, Piscataway, NJ, 1986).

2 P. M. Meaney, K. D. Paulsen, and J. T. Chang, "Near-field microwave imaging of biologically-based materials using a monopole transceiver system," IEEE Transactions on Microwave Theory \& Techniques 46, 31-45 (1998).

3 R. G. Olsen and J. C. Lin, "Acoustic imaging of a model of a human hand using pulsed microwave irradiation," Bioelectromagnetics 4, 397-400 (1983).

4 R. L. Nasoni, G. A. Evanoff Jr., P. G. Halverson, and T. Bowen, "Thermoacoustic emission by deeply penetrating microwave radiation," Proc. IEEE Ultrasonics Symposium 5, 633-637 (1984).

5 K. H. Chan and J. C. Lin, "Microwave-induced thermoacoustic tissue imaging," Proc. Engineering in Medicine and Biology Society 10th Annual International Conference, 445-446 (1988).

6 R. A. Kruger, K. K. Kopecky, A. M. Aisen, D. R. Reinecke, G. A. Kruger, and W. L. Kiser Jr., "Thermoacoustic CT with radio waves: a medical imaging paradigm," Radiology 211, 275-278 (1999).

7 L.-H. Wang, X. Zhao, H. Sun, and G. Ku, "Microwave-induced acoustic imaging of biological tissues," Rev. Sci. Instrum. 70, 3744-3748 (1999).

8 G. Ku, L.V. Wang, "Scanning thermoacoustic tomography in biological tissue," Med. Phys., in press (2000)

9 N. Bronshtein, K. A. Semendyayev, Handbook of Mathematics (Van Nostrand Reinhold Company, New York, 1978).

10 G. J. Diebold, and T. Sun, "Properties of photoacoustic wave in one two and three dimensions," Acoustica, 80 339-351 (1994). 